\title{
Subject to change
}

\section{Getting to grips with scientific variables.}

\begin{abstract}
Joseph Lachance
Huang, L. et al. Comments re: Lee, V. et al. Science 322, 123 (2009)

"All efforts to repeat the results by Lee $\mathrm{et}$ al. have been unsuccessful. While it is understandable that they lack our impeccable experimental technique, we believe that they failed to properly control for..."
\end{abstract}

Jones, M. C. Irreproducibility. Philos. Sci. 78, 143-151 (2011).

"An increasing number of studies show a lack of reproducibility. In addition, accusations of scientific fraud have skyrocketed in the past year. Most famously, the dry-ice bomb incident between chemists at Oxford and Chicago led to no less than 7 arrests..."

Jimenez, F. Physical laws are local. Nature 482, 432-435 (2014).

"Research sites on either side of the Wallace Line consistently obtain different results. These experiments conclusively demonstrate that physical laws are not the same in different locations. It has not escaped our attention that this phenomenon may be responsible for numerous discrepancies in the past 5 years $^{9-17}$. We have no choice but to conclude: what happens in Vegas, stays in Vegas..."

Lapointe, A. The End of science? Time 186, 24-31(2015).

“This is not your father's or mother's physics. At a recent conference in Zurich, physicists heatedly debated the objectivity of science. If physical laws do indeed vary from location to location, the foundations of science are on shaky ground. Matters were not helped when it was revealed that a cultural relativist accepted the Ultimate Boeing 747 gambit, only to glide safely and slowly down to earth from a height of 30,000 feet. Science as defined by Karl Popper is dead..."

Orbach, R. et al. Aether or dark matter?

Astrophys. J. 814, 78-90 (2016).

"Signals from the pulsar J1342+2822B seem to have been modified in transit. Results are consistent with the effect of a gravitational lens, perhaps due to the existence of dark matter. However, an alternative explanation is that the signal passed through a region of space with different physical laws. If so, this would mean that the Jimenez effect is more pervasive than previously thought..."

Kabaka, F. et al. Evidence of dynamic physical laws. PLoS Phys. 6, 1-17 (2017).

"Results from supercollider experiments exhibited a marked change after 09:43 GMT on 12 January 2017. Something clearly changed. Prior to 12 January, readings at CERN were $9.34 \pm 0.3 \mathrm{MeV}$. Subsequent readings were $5.34 \pm 0.2 \mathrm{MeV}$. Interestingly, readings at Fermilab were consistently in the $9.34 \mathrm{MeV}$ range. This suggests that physical laws are both local and dynamic..."

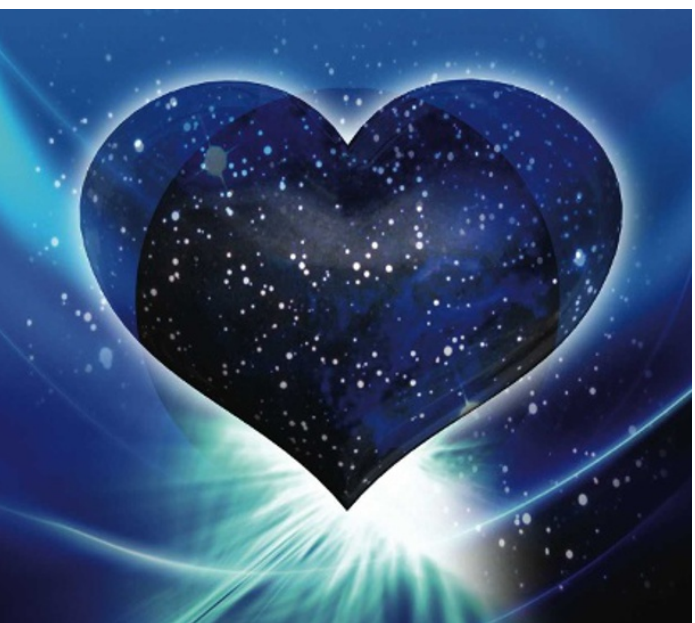

Singh, N. A history of cellular automata. Phys. Rev. Lett. 118, 56-72 (2017).

"Furore over dynamic physical laws has led to increased interest in cellular automata and artificial life. Cellular automata are spatial models in which states of cells within a grid are updated in discrete steps. Simple spatial rules, such as those of Conway's Game of Life, can generate complex results. Here, the state of a cell depends on the states of neighbouring cells. Patterns such as 'blocks', 'blinkers' and 'gliders' have been found. Some patterns give the appearance of being alive. It is rumoured that the amount of computing time dedicated to the Game of Life is exceeded only by SETI@Home and World of Simcraft...”

Chen, X.\& Li, H. Meta-rules and the search for universality. Preprint at <http://arxiv.org/ abs/0992.4174> (2019).

"Recent discoveries have divided the scientific community into two warring camps. Localists believe the new paradigm that physical laws are local, whereas Universalists cling to the idea that physical laws are universal. We suggest that local laws are determined by some universal meta-rule. This could bring the Localist-Universalist feud to an end, allowing resources to be diverted to empirical studies of Murphy's law (a research programme that has garnered such prestigious awards as the IgNobel prize) ..."

Williams, C. et al. Physical Laws are Cellular Automata. Proc. Natl Acad. Sci. USA 116, 78-92 (2019).

"As shown in Figure 3, local laws are spatially and temporally correlated. Laws belong to a finite number of configurations and depend on laws present at nearby locations. This indicates that physical laws behave as cellular automata. Patterns of physical laws exhibit both periodicity and mobility. Interestingly, 'Rule 110 ' seems to govern the behaviour of fringe elements..."

\section{Abel, C. \& Wilkins, O. Evolution of} simulated organisms. Artif. Life 26, 231-239 (2020).

"Artificial life can exist in the form of cellular automata. Using a set of iterated, spatial rules, we simulated an ecosystem on a large cluster of microcomputers. For the first time, the existence of a virtual organism more complex than Escherichia coli has been demonstrated (see supplemental pages S1-S23 for definitions of complexity). It remains to be seen if sentience can evolve in silico..."

Meme, A. Life within the laws of the Universe. www.stochasticity.scienceblogs.com (2021).

"If I understand things correctly, this means that life, or something like it, exists on a truly grand scale. No, the Universe is not a giant game of Go. Instead, the rules of the Universe are flickering between states as if they are cellular automata. The bizarre events of the past few years seem to fit with a 'glider' passing through our local neighbourhood. Things have returned to normal, but for how long? If 'gliders' can exist, so too might other more complex patterns. Does this mean that the Universe itself is alive? I can only begin to wonder what strange organisms exist within the fabric of the Universe. Perhaps we are not alone!..." Joseph Lachance is a PhD student at Stony Brook University, where he studies theoretical population genetics and Drosophila genetics. 